\title{
Design and Implementation of the Multi-parameter Laser Test System
}

\author{
H.W. Yang \\ Baicheng Ordnance Test Center \\ China
}

\author{
Y.H. Shan \\ Baicheng Ordnance Test Center \\ China
}

\begin{abstract}
In order to automatically measure the coordinates of the bullets impacting with high degree of accuracy and in noncontact way, we designed a multi-parameter laser test system, of which operating principle, structure and composition, and design points are elaborated in the paper. This system can accurately measure such parameters as bullet flight time, velocity, angle of jump, etc. The corresponding test method in the paper is tried and tested. The system has more broad application prospect in shooting training and appraisal in the army and other departments.
\end{abstract}

\section{Keywords-laser target; system design; parameter testing}

\section{INTRODUCTION}

It is necessary to measure the coordinate of the bullet impacting when firing at some distance target, whether in evaluating the weapon accuracy or checking the shooting level of a weapon user. As manual measurement is inefficiency, in addition, there is a certain security risk in using it, the automatic target-reading system is widely equipped in firing range now. However, in some cases that require high accurate coordinate, these present mainstream automatic target-reading systems (e.g. acoustics target, light-screen target, CCD target, and so on) can not fully meet the need[1][2][3]. The multiparameter laser test system, as described in the paper, measures the coordinate of the bullet impacting with the orthogonal laser-screen produced by low power semiconductor laser arrays. This system has such advantages as high measuring accuracy, reliable work and multi-parameter measurement when compared with its kind.

\section{SYSTEM DESIGN AND IMPLEMENTATION}

\section{A. System Configuration and Operating Principle}

The multi-parameter laser test system consists of rectangular coordinate type laser target subsystem, data acquisition and transmission subsystem, data processing subsystem and infrared detector. Figure I shows the principle diagram of the system.
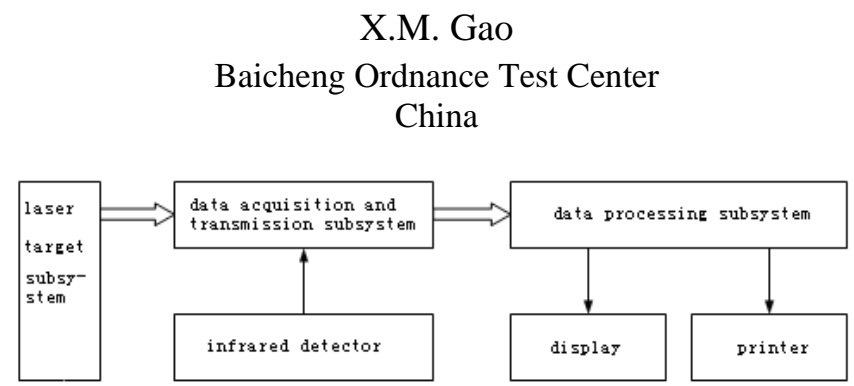

FIGURE I. PRINCIPLE DIAGRAM OF THE SYSTEM

The operating principle of measuring the coordinates of the bullets impacting with the system is: the muzzle flame appears when the bullet is coming out of the barrel; if the muzzle flame is detected by the infrared detector, a firing signal is produced and transmitted to data acquisition and transmission subsystem; as soon as the acquisition and transmission circuit receives the firing signal, it turns on data acquisition gate circuit under the control of single-chip microcomputer, and begins to collect data. When the bullet is passing through the target surface in some position, photosensor in corresponding coordinate provide bullet impacting signal, which is preprocessed by the acquisition and transmission circuit, and is transmitted to data processing subsystem according to communication protocols. Data processing subsystem processes (calculates, displays, and stores) each bullet data received, and exports the result in report form of requirements.

\section{B. Design and Implementation of the System Structure}

1) Design of the mechanical structure of the system

In order to achieve the purpose of use, the whole system is divided into two parts in engineering, i.e. front end (target position) and back end (gun position).

The laser target subsystem, the data acquisition and transmission subsystem are integrated into the test target frame in the front end. The test target frame is fixed in the special rack when used. It requires protective shields to be set in front of the device and in direction of the bullet coming in case individual bullet should strike the test target frame when firing. Moreover, it requires the aiming device for the weapon user. Figure II shows the components of the system front end.

The main part of the back end of the system is data processing subsystem. The two parts communicate with each other through the multi-core cable or the micro-power radio station. 


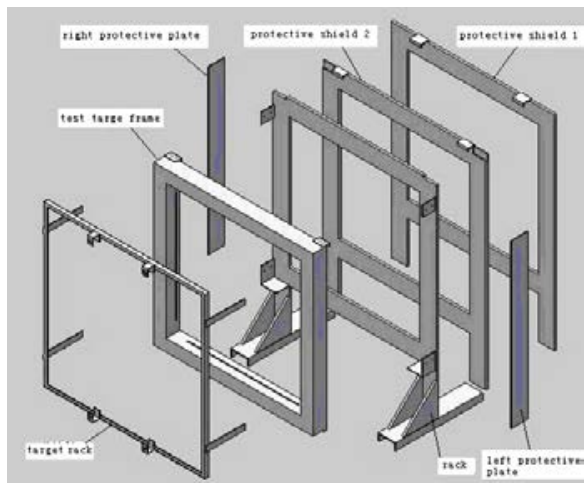

FIGURE II. SCHEMATIC DIAGRAM OF THE FRONT-END STRUCTURE.

2) Design of the system circuit

The system circuit consists of the shaping amplifier circuit for high frequency small signal, the high-speed data acquisition and coding register circuit, the controlling circuit and the single-chip microcomputer control software (solidified in the microcontroller chip).

(a) Design of the shaping amplifier circuit for high frequency small signal

As the laser beam has the characteristics of being prone to be collimated, concentrated energy and being fast in photovoltaic conversion, that using the single amplifier circuit can effectively improve the anti-jamming capability of the device lest multi-stage amplifier circuit for small signal be susceptible to disturbance. After the signal amplified, it is shaped into standard TTL pulse signal. FigureIII shows the schematic diagram of the circuit.

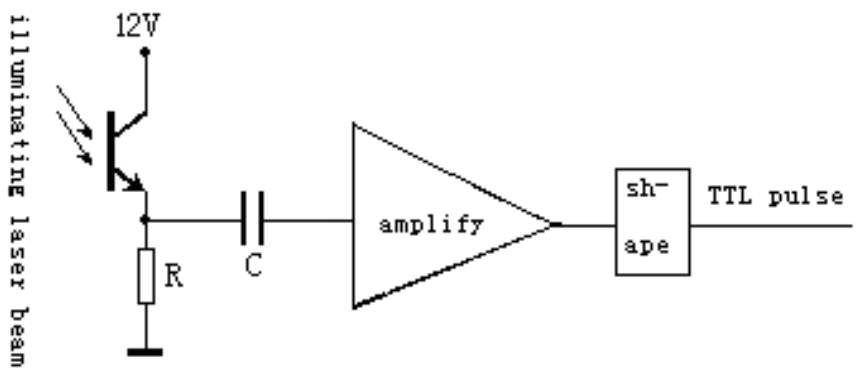

FIGURE III.

THE PHOTOVOLTAIC CONVERSION AND SHAPING AMPLIFIER CIRCUIT.

(b) Design of the high-speed data acquisition and coding register circuit

In order to ensure that the system can be applied to various types of small arms, the effective measurement area of the front part of the system should be large enough, which results in many measurement signals and large data. Hence, it is necessary to use the high-speed data acquisition, storing and transmission circuit. In order to reduce the circuit scale and improve system reliability, the coded signal is used. One group is composed of every 8-channel signals and one bit composed of signals from the same channel of every group. With the 8group signals and 8-bit signals, we can determine the exact location of every channel signal in 64 channels. Every 64channel signals constitute a section (there are several sections in $\mathrm{Z}$ or $\mathrm{Y}$ direction). Every section is assigned a address code. According to the address code, the group signal and the bit signal, the exact location of every channel signal in the whole target surface can be determined. A section code and the circuit logic diagram can be seen in figureIV.

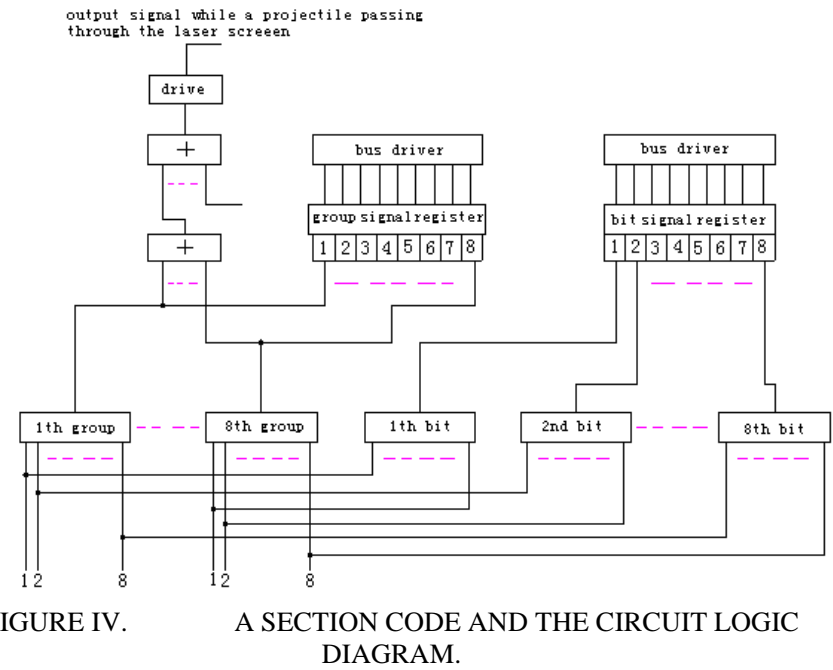

Duo to the limited space, we do not repeat the other circuit design.

3) Key points of the system software design

The system software design should depend on the customer requirements that measuring different parameters. The system software consists of the management and control software and the client software. As it is possible that some laser test systems are equipped in a shooting range, the management software can manage, control permission and print data report for one or all of the test devices. The client software can be operated by the weapon user who can choose test parameter, input user name and weapon type, and so on.

\section{Multi-Parameter Test Method}

Through proper combination, the multi-parameter laser test systems can be used in measuring bullet flight speed, flight time, angle of jump of a firearm, shooting frequency, etc., in addition to the coordinate of the bullet impacting.

Using multiple multi-parameter laser test systems and multi-channel chronographs, we can measure bullet flight speed and flight time. Figure $V$ is the schematic diagram of the test system. There are five laser test systems in the figure. This system can provide bullet speed of $5 \mathrm{~m}$ and $25 \mathrm{~m}$ from the muzzle while measuring the flight time.

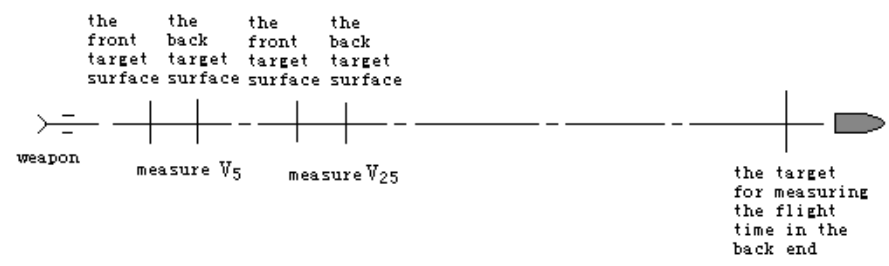

FIGURE V. THE SCHEMATIC DIAGRAM OF MEASURING BULLET FLIGHT TIME. 
We can use a single system or a double-system when measuring the angle of jump of a firearm. The two methods have their own advantages. The following analysis focuses on this issue.

1) The method on measuring the angle of jump with single system

This method is same as the traditional one which uses paper target in measuring the angle of jump: lay the laser test system in $50 \mathrm{~m}$ from the muzzle ( handgun, $20 \mathrm{~m}$ ); adjust the origin in the longitudinal axis of the test target frame to the same height as the muzzle horizontal with a leveling instrument or a transit instrument; keep the aiming line horizontal as possible. To see the specific situation, refer to Figure VI[4].

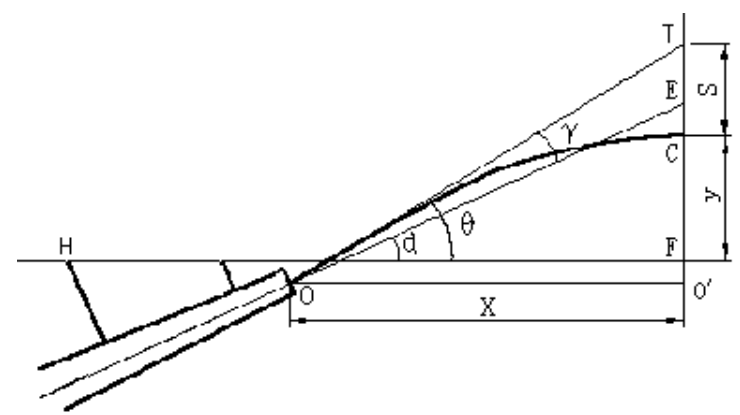

FIGURE VI. THE SCHEMATIC DIAGRAM OF MEASURING THE ANGLE OF JUMP WITH SINGLE SYSTEM.

The longitudinal coordinate $\mathrm{y}$ and the bullet flight time from the muzzle to the target can be obtained directly by using the laser test system. There are two methods on calculating the angle of jump.

Method one: at the first, average $\mathrm{y}$ and $\mathrm{t}$ of a group of bullets, then calculate the angle of jump $\gamma$. The formula is :

$$
\begin{aligned}
& \bar{y}=\frac{1}{n} \sum_{i=1}^{n} y_{i} \\
& \bar{t}=\frac{1}{n} \sum_{i=1}^{n} t_{i} \\
& \bar{\theta}=\left(\frac{\bar{y}+\frac{1}{2} g \bar{t}^{2}}{X}\right) \rho \\
& \bar{\gamma}=\bar{\theta}-\alpha
\end{aligned}
$$

Method two: first, calculate the angle of jump of each bullet; then, calculate the average angle of jump of a group of bullets. The formula is:

$$
\theta_{i}=\left(\frac{y_{i}+\frac{1}{2} g t_{i}^{2}}{X}\right) \rho
$$

$$
\begin{aligned}
& \gamma_{i}=\theta_{i}-\alpha \\
& \bar{\gamma}=\frac{1}{n} \sum_{i=1}^{n} \gamma_{i}
\end{aligned}
$$

2) The method on measuring the angle of jump with double systems

The measuring process is: lay the $1 \#$ target in $3 \sim 5 \mathrm{~m}$ from the muzzle; lay the $2 \#$ target in $50 \mathrm{~m}$ from the $1 \#$ target; adjust the origins in the longitudinal axes of the two targets to the same height with a leveling instrument, or precisely measure the height difference between the two origins; keep the aiming line horizontal as possible. The main benefit of using the double systems is that the origin height of the longitudinal coordinate is not limited by the muzzle height, hence, it can be adjusted according to the situation in the test field. The specific conditions are shown in FigureVIII.

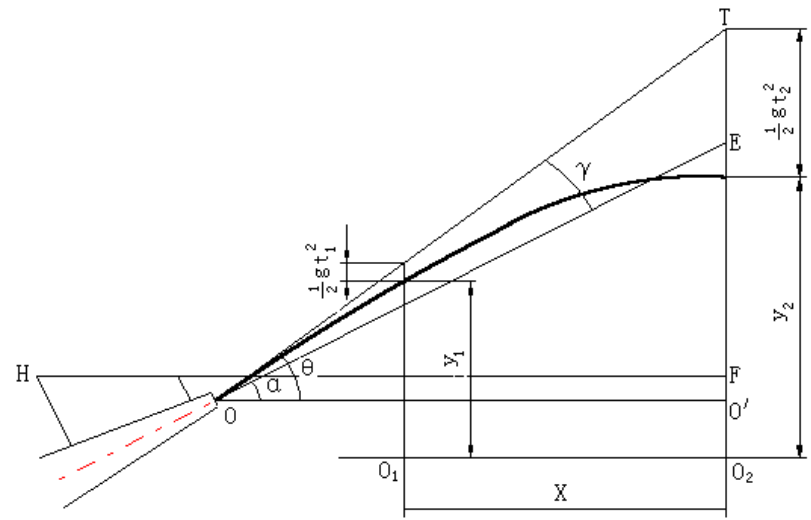

FIGURE VII. THE SCHEMATIC DIAGRAM OF MEASURING THE ANGLE OF JUMP WITH DOUBLE SYSTEMS.

The basic measuring principle of the double systems test is same as that of the single system test. The formula of calculating the angle of jump is:

$$
\begin{gathered}
\gamma=\theta-\alpha \\
\theta=\frac{\left(y_{2}-y_{1}\right)+\frac{1}{2} g\left(t_{2}^{2}-t_{1}^{2}\right)}{X} \rho
\end{gathered}
$$

There are also two methods on calculating the average angle of each group.

Method one:

$$
\bar{\gamma}=\frac{\left(\bar{y}_{2}-\bar{y}_{1}\right)+\frac{1}{2} g\left(\bar{t}_{2}^{2}-\bar{t}_{1}^{2}\right)}{X} \rho-\alpha
$$

Parameter $\bar{y}$ and $\bar{t}$.can be calculated according to equation (1) and (2).

Method two: 


$$
\gamma_{i}=\frac{\left(y_{2_{i}}-y_{1_{i}}\right)+\frac{1}{2} g\left(t_{2_{i}}{ }^{2}-t_{1_{i}}{ }^{2}\right)}{X} \rho-\alpha
$$

The average angle of jump can be calculated according to equation (7).

\section{CONCLUSIONS}

The core technology of the multi-parameter laser test system is using the orthogonal laser-screen produced by low power semiconductor laser arrays to realize non-contact measuring the coordinate of the bullet impacting. With the combination of some systems, the multi-parameter laser test systems can also realize non-contact measuring bullet flight time, flight speed and angle of jump of a firearm, etc. Through the practical validation, the system can meet the need of evaluating weapon firing accuracy and acquiring the parameters in firing table. The system has been well applied a certain number of weapons research and development units. The system will have a broad application prospect in test range, research institutes, arsenal or force, public security system, and so on, which often carry out shooting training and assessment.

\section{REFERENCES}

[1] Austria HIGH PRESSURE INSTRUMENTATION.B541 Acoustic Target System Operating Instruction.1998

[2] Austria HIGH PRESSURE INSTRUMENTATION.B571 Optical Target System Operating Instruction.1998

[3] xi'an institute of optics and precision mechanics of cas. The preresearch summary report on coordinate automatic measurement system for CCD target of small arms.2001.

[4] D.B. Li, S.H. Guo, GJB349.6-87 conventional weapon approval test method, preparation of the firearms firing table [S].1987. 